\title{
Surto de malária induzida entre usuários de drogas injetáveis
}

\section{Outbreak of malaria among injectable-drug users}

\author{
Luiz Carlos Barradas Barata *, Maria Tereza M. Andriguetti *, Marina Ruiz de Matos *
}

\begin{abstract}
BARATA, L. C. B. et al. Surto de malária induzida entre usuários de drogas injetáveis. Rev. Saúde Pública, 27: 9-14, 1993. Em julho de 1990, foi registrado na cidade de Bauru, Estado de São Paulo, Bràsil, um surto de malária envolvendo usuários de cocaína injetável. Uma ampla investigação epidemiológica, conduzida de 19 de julho a 13 de setembro, revelou que pelo menos 119 pessoas estavam envolvidas no surto, uma vez que haviam compartilhado seringas e agulhas com um ou mais casos confirmados nos 3 meses anteriores à ocorrência. Cento e duas dessas pessoas foram localizadas e entrevistadas, e destas, 99 foram submetidas a exame de gota espessa e 91 a exames sorológicos para malária. Foram confirmados por exame hemoscópico 21 casos de malária por $P$. vivax, e 3 outros tiveram exame sorológico positivo para $P$. vivax. O controle da transmissão foi obtido fornecendo-se cloroquina aos envolvidos no surto, numa dose inicial de 10 comprimidos, seguida de doses supressivas semanais de 2 comprimidos até que fosse identificado o último comunicante. Amostras de soro coletadas na ocasião revelaram, ao lado da malária, uma alta prevalência de infecções pelo HIV $(58 \%)$ e pelo vírus da hepatite B $(40 \%)$. Foram discutidas as dificuldades para o controle do surto e a possibilidade da malária vir a se tomar uma doença endêmica entre usuários de drogas injetáveis, no Estado de São Paulo.
\end{abstract}

Descritores: Malária, epidemiologia. Plasmodium vivax. Abuso de substâncias por via intravenosa, complicações. Uso comum de agulhas e seringas.

No Estado de São Paulo a malária é uma doença de notificação compulsória e a Superintendência de Controle de Endemias (SUCEN) é o órgão responsável pelo seu controle, através da confirmação laboratorial, investigação epidemiológica e tratamento dos casos, e combate aos surtos autóctones da doença.

Na cidade de Bauru, a SUCEN conta com um laboratório de malária que atende 30 municípios vizinhos. De 1985 a 1989, 641 casos de malária foram diagnosticados na região sendo todos eles importados.

O município de Bauru situa-se na região oeste do Estado de São Paulo; a 324 km da cidade de São Paulo, e contava em 1990 com uma população estimada de 260.000 habitantes, $98 \%$ deles morando na área urbana. A cidade é um importante entroncamento rodo-ferroviário integrando as fronteiras do Estado com a Amazônia brasileira.

No dia 6 de julho de 1990 um caso de malária

\footnotetext{
* Superintendência de Controle de Endemias - SUCEN São Paulo, SP - Brasil.
}

Separatas/Reprints: L. C. B. Barata - Rua Paula Souza, 166 - $1^{\circ}$ andar - 01027-000 - São Paulo, SP - Brasil.

Ediçāo subvencionada pela FAPESP. Processo Medicina 93/ 0208-5. por $P$. vivax foi notificado pelo Hospital de Base de Bauru, à SUCEN. A investigação epidemiológica do caso revelou que o paciente nunca havia tido malária, não havia se deslocado por áreas endêmicas e não recebera transfusão de sangue ou derivados. Como o paciente negasse também o uso de drogas injetáveis, o caso foi considerado inicialmente como autóctone, embora desde 1978 não houvesse registro de autoctonia na região.

Nos dias 14 e 16 do mesmo mês, dois outros casos foram notificados pelo Pronto-Socorro Municipal de Bauru e, no dia 17, dois outros pacientes procuraram espontaneamente o laboratório da SUCEN no município. Estes dois pacientes, quando entrevistados, declararam que faziam uso de cocaína injetável compartilhando seringas e agulhas. Posteriormente, durante uma nova entrevista, acrescentaram que haviam sido alertados sobre a possibilidade de malária pelo caso identificado no dia 6 de julho, com quem haviam feito uso de droga injetável recentemente.

Diante destas informaçōes ficou evidente que se tratava de um surto de malária induzida entre usuários de drogas e, a SUCEN, em conjunto com a Secretaria Municipal de Saúde de Bauru e o Escritório Regional de Saúde local, passou a desenvolver um trabalho de investigação epidemiológica em larga escala na cidade, que é o objeto do presente trabalho. 


\section{A Estratégla da Investigaçáo e Controle do Surto}

As atividades especificas de controle adotadas tiveram início em 19 de julho de 1990, quando 5 casos já haviam sido diagnosticados.

$O$ processo de investigação do surto partiu de informaçōes prestadas pelos primeiros casos detectados, aos quais se solicitou que fornecessem informaçס̃es sobre seus comunicantes, o que possibilitou a constituição de uma relação inicial de suspeitos. Estes indivíduos foram visitados em suas casas e informados sobre a ocorrência de malária. A seguir foi-lhes apresentada a relação de suspeitos na qual, evidentemente, estavam relacionados seus próprios nomes e solicitado que indicassem os individuos com quem haviam compartilhado seringas e agulhas nos últimos 3 meses e, se fosse o caso, fornecessem informaçð̃es sobre outros comunicantes que porventura não contassem da lista. Nessas visitas procedia-se à coleta de amostra sangüínea para realização de exame hemoscópico de malária de todos os indivíduos, com ou sem sintomas da doença.

Repetindo-se esse procedimento, foi possível constituir uma relação de suspeitos, considerados como tais os indivíduos que nos 3 meses precedentes haviam feito uso de droga injetável com pelo menos um caso confirmado laboratorialmente. Foram considerados casos confirmados apenas os que tiveram exame hemoscópicos realizado pela técnica da gota espessa positivo para malária.

Todos os casos confirmados ou suspeitos que puderam ser localizados foram investigados com um questionário padronizado no qual constavam perguntas sobre antecedentes maláricos, viagens nos últimos 18 meses por áreas endêmicas de malária, transfusões sangüíneas nos últimos 3 meses, uso de drogas injetáveis e sintomas de malária.

O tratamento proposto para os casos confirmados e suspeitos foi cloroquina numa dose inicial de $1.500 \mathrm{mg}$ ( $600 \mathrm{mg} 1^{2}$ dia e $450 \mathrm{mg}$ nos 2 dias restantes do tratamento) imediatamente após a coleta de sangue destinada à hemoscopia, seguido de 300 $\mathrm{mg}$ de cloroquina semanalmente até que o último caso suspeito fosse investigado. Esta indicação foi feita mesmo para aqueles que não apresentavam sintomas. Foram coletadas amostras de sangue para realização de exame sorológico para malária pela técnica de imunofluorescência indireta (RIFI) com antígenos de $P$. vivax e $P$. falciparum.

Amostras de soro foram também testadas para a presença de antígenos do vírus da hepatite $B$ $\left(\mathrm{AgHB}_{\mathrm{s}}\right)$ pelo método de ELISA e anticorpos contra doença de Chagas, sífilis e HIV, pelos métodos de imunofluorescência indireta, VDRL e ELISA, respectivamente.

Todas as informações foram obtidas mediante a garantia de que seriam mantidas anônimas, sob sigilo, e utilizadas apenas com a finalidade de controlar o surto.

Paralelamente ao trabalho de visitas domiciliares, foi realizado em Bauru uma ampla divulgação junto aos órgãos de saúde e à imprensa local sobre a natureza da ocorrência, buscando alertar os possíveis implicados sobre a importância de procurarem prontamente assistência médica.

O trabalho de visita domiciliar se estendeu de 19 de julho a 13 de setembro de 1990, quando foi identificado o último caso suspeito.

\section{Resultados das Atividades de Investigaçáo e Controle}

Através das atividades desenvolvidas em Bauru, 119 casos suspeitos de malária foram relacionados, sendo que 102 deles foram investigados (12 casos não puderam ser localizados e 5 recusaram receber os serviços de saúde). Foram realizados exames hemoscópicos de 99 casos suspeitos (houve 3 recusas), resultando em 21 casos confirmados com hemoscopia positiva para $P$. vivax.

A distribuição por sexo e idade dos casos confirmados e suspeitos revelaram uma preponderância de indivíduos do sexo masculino $(72,5 \%)$, com a idade variando de 10 a 44 anos (idade média de 23 anos, desvio padrão 6,3). Na Tabela acham-se discriminadas as ocupaçסes exercidas por esse grupo de pessoas no momento da investigação. Foram destacadas prostitutas em função do potencial de transmissão de outras doenças e vendedores viajantes por se constituírem pessoas

Tabela. Distribuição dos casos confirmados e suspeitos de malária induzida entre usuários de droga injetável de acordo com o tipo de ocupação exercida. Bauru, 1990.

\begin{tabular}{lrr}
\hline Ocupação & Ne & $\%$ \\
\hline - Trabalhadores manuais sem qualificação** & 12 & 11,7 \\
prostitutas & 06 & 5,9 \\
- Trabalhadores qualificados de nível básico e & 27 & 26,5 \\
médio** & 23 & 22,5 \\
Vendedores, viajantes & 02 & 2,0 \\
- Trabalhadores especializados de nivel uni- & 32 & 31,4 \\
versitário & 102 & 100,0 \\
- não trabalham*** & & \\
\hline Total & & \\
\hline * Inclui serventes de pedreiro, lavadores de carros, \\
trabalhadores rurais, etc. & & \\
** Inclui carpinteiros, cozinheiros, bancários, etc. \\
*** Inclui estudantes, donas de casa e desempregados.
\end{tabular}


de alta mobilidade, mantendo deslocamentos rotineiros para diversos municípios do Estado.

A duração do surto foi de 51 dias. Na Figura encontra-se a distribuição, no tempo, dos casos confirmados e suspeitos de acordo com a data de identificação dos mesmos.

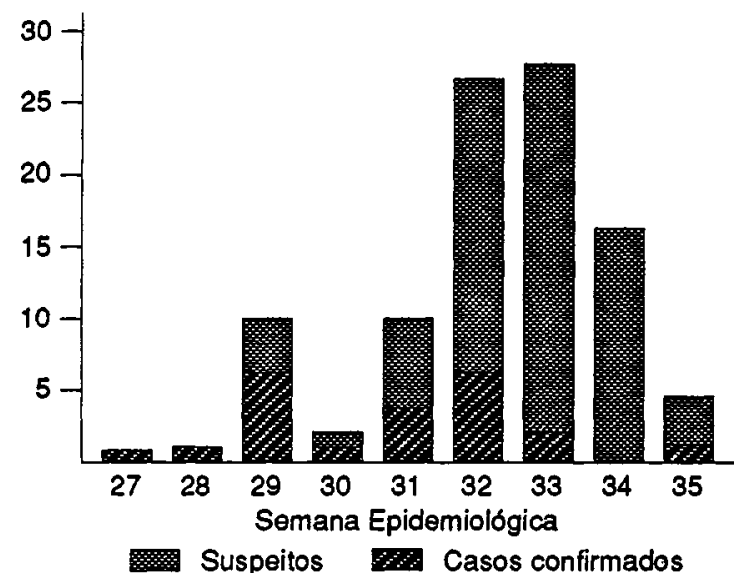

Flgura. Distribuiçăo dos casos confirmados e suspeitos de malária induzida, submetidos a exames hesmoscópicos. Fonte: SUCEN.

Dentre os indivíduos investigados foram identificados grupos cujas relaçōes, além das propiciadas pelo uso coletivo de cocaína injetável, eram de parentesco e vizinhança. A distribuição geográfica dos casos confirmados e suspeitos mostrou a proximidade de grande número deles, localizados em bairros periféricos da cidade, indicando um tipo de contrato entre eles quase que setorizado. Esses grupos, entretanto, não eram fechados em si. Alguns indivíduos relataram se utilizar-se da compra da aplicação da droga em locais freqüentados por pessoas oriundas de bairros diversos da cidade. Nesses locais um número de usuários se cotizava na compra de uma determinada quantidade de droga que era aplicada pelo próprio fornecedor.

O número de parceiros na utilização de seringas e agulhas variou de 1 a 20 ou mais, nos 3 meses anteriores à identificação dos casos. Das 102 pessoas entrevistadas, $59(57,8 \%)$ mencionaram de 1 a 5 parceiros; $20(19,7 \%)$, referiram de 6 a mais de 20 e $23(22,5 \%)$ negaram compartilhar seringas e agulhas apesar de terem seus nomes indicados por pelo menos um caso confirmado.

A investigação epidemiológica revelou que a maior parte dos envolvidos no surto não havia viajado para áreas endêmicas de malária nos 18 meses anteriores à detecção do caso inicial, à exceção de 4 indivíduos assintomáticos e com exames hemoscópicos negativos que relataram deslocamento para a Amazônia (um desses casos teve RIFI positiva para $P$. vivax, com título igual a 1:64).

Nenhum dos 102 casos investigados relatou episódio anterior de malária.

De 90 casos investigados de que foram obtidas informaçð̃es acerca de sintomalogia sugestiva da malária (febre precedida de calafrios e tremores), $63(70 \%)$ negaram sua ocorrência e $27(30 \%)$ referiram acessos febris característicos. Dos 27 sintomáticos, 21 se automedicaram com antibióticos após o início dos sintomas. Entre os 21 casos confirmados hemoscopicamente, $19(90,5 \%)$ relataram sintomas de malária, 13 dos quais com acessos febris cotidianos.

Durante as atividades de controle do surto, 99 pessoas ( $97 \%$ dos casos investigados) foram medicadas com uma dose inicial de cloroquina de 1,5 gr (3 indivíduos com hemoscopia negativa recusaram o tratamento), e 58 foram mantidas com dose supressivas semanais do medicamento, o que correspondeu a uma cobertura de $64 \%$ (11 individuos não chegaram a receber medicação supressiva pois foram detectados nos 7 dias anteriores à identificação do último suspeito).

$O$ intervalo do tempo transcorrido entre o início dos sintomas e o diagnóstico de malária nos 21 casos confirmados foi sempre superior a 15 dias (5 entre 16 a 29 dias, 8 entre 30 a 59 e 8 entre 60 a 150 ).

Amostras de soro foram coletadas de 91 pessoas, sendo que 21 apresentaram resultados positivos quando testadas com antígenos de $P$. vivax, considerando diluiçōes significativas aquelas iguais ou superiores a 1:32. Quando comparados aos resultados da hemoscopia, observaram-se 18 casos confirmados por ambas as técnicas e $3 \mathrm{com}$ apenas exame sorológico positivo (3 casos confirmados hemoscopicamente recusaram a coleta de amostra sanguiínea). Com relaçāo às demais doenças estudadas, obteve-se positividade das amostras para sífilis (12\%), hepatite B (40\%) e AIDS (58\%). Nenhuma amostra resultou positiva para doença de Chagas ou $P$. falciparum.

\section{Discussăo}

Casos de malária entre usuários de drogas injetáveis foram descritos pela primeira vez por Biggan $^{2}$, no Cairo, Egito, em 1929. O modo de transmissão nessa circunstância foi descrito de maneira detalhada por Biggan ${ }^{2}$, Eaton \& Feinberg 4 e por Most $^{10}$. O compartilhamento de seringas, agulhas e outros aparatos destinados à preparação e administração endovenosa das drogas resulta na contaminação desses equipamentos com sangue o que possibilita a disseminação de várias doenças, dentre elas a malária. 
Lo e col. ${ }^{8}$ descreveram pela primeira vez no Estado de São Paulo a transmissão de malária entre 8 usuários de cocaína injetável na cidade de Presidente Prudente, São Paulo, nos anos de 1988 e 1989. No ano de 1989, 2 casos foram também registrados na cidade de São Paulo ${ }^{1}$. O surto ocorrido na cidade de Bauru, em 1990, relatado no presente trabalho, foi o maior observado até o momento e mereceu atenção especial dos serviços de saúde devido ao grande número de pessoas envolvidas.

A ocorrência de malária entre usuários de drogas endovenosas, detectadas pela primeira vez no Estado de São Paulo, no final da década de 80, pode ser explicada pela intensificação da transmissão natural da doença na Amazônia de onde provém a quase totalidade dos casos registrados no Estado e pelo número significativo de usuários de drogas injetáveis presumivelmente existentes nesse Estado.

Na Amazônia, o número de exames positivos para malária subiu de 169.871, em 1980, para 560.396 , em 1990, sendo a transmissão especialmente intensa nos assentamentos rurais recentes e nos garimpos $9,13,15$. Nestes últimos, segundo depoimento de vários pacientes atendidos pela SUCEN, o comércio e o consumo de drogas entre os trabalhadores não é raro.

Com relação ao uso de drogas injetáveis no Estado de São Paulo, embora não existam informações oficiais sobre 0 assunto, os dados relativos à AIDS indicam que, de fato, o número de usuários deve ser consideravelmente grande. Em $1990,34,5 \%$ dos casos de AIDS identificados no Estado estavam relacionados ao uso de drogas injetáveis ${ }^{12}$.

A origem do surto de malária em Bauru não pôde ser completamente esclarecido. A observação, entretanto, de que pelo menos 4 indivíduos haviam frequientado garimpos ou outras áreas da Amazônia, num período recente antes da deteç̧ão do primeiro caso, e de que um deles apresentava reação de imunofluorescência indireta (RIFI) positiva para $P$. vivax, sugere que um ou mais deles possam ter sido os responsáveis pela introdução da doença.

A estratégia adotada para se identificar os suspeitos baseou-se no trabalho descrito por Friedmann e col. ${ }^{5}$ para o controle de um surto semelhante ocorrido em Bakersfield, Califórnia (USA), em 1970-71. Em Bauru, a concentração dos casos em alguns poucos bairros da cidade facilitou a identificação e localização das pessoas envolvidas no surto, em que pese a marginalidade e a grande mobilidade de muitos deles.

$O$ fundamento da estratégia utilizada consiste na identificação e tratamento de todos os envolvidos no surto, independentemente da presença de sintomas sugestivos da doença ou da confirmação laboratorial da infecção, uma vez que alguns deles podem estar no período de incubação e/ou com parasitemia subpatente no momento da coleta da amostra sanguínea inicial, e a repetição dos exames hemoscópicos é na maioria das vezes impraticável devido a dificuldades de localização e abordagem desses indivíduos.

A RIFI pouca contribuição fornece neste sentido também. Resultados positivos aparecem 7 a 12 dias após infecção, geralmente logo após o início dos primeiros sintomas ${ }^{17}$. Embora diluições superiores a 1:64 reflitam comumente uma resposta imune à infecção, não necessariamente indicam a presença de parasitas no sangue. Utilizando antígenos de $P$. falciparum, diferentes autores sugerem diluições de 1:20 a 1:40 para fins de exclusão de doadores de sangue por indicarem a possibilidade de infecção sangúínea presente ${ }^{17}$. Considerando títulos iguais ou superiores a 1:32 como significativos, os resultados dos exames sorológicos realizados em Bauru sugerem que pelo menos 3 outras pessoas possam ter sido infectadas embora não tenham apresentado exames hemoscópicos positivos, o que reforça a idéia de que se deva tratar os casos suspeitos independentemente do resultado da hemoscopia.

Dentre os casos descritos por Lo e col. ${ }^{8}, 3$ reinfecç̋es foram observadas, sendo duas delas num mesmo paciente, o que indica que usuários de drogas injetáveis não costumam alterar seus hábitos de compartilhar seringas e agulhas, mesmo após saberem do risco de contrair malária por essa prática. Para se evitar a reinfecção, e a infecção, num futuro próximo, de outras pessoas ainda não infectadas, optou-se, na presente investigação, pelo fornecimento de cloroquina em doses supressivas semanais para todos os individuos, após o tratamento inicial. Embora a cobertura alcançada com essa medida não tenha sido ideal, ela parece ter sido importante para a não ocorrência de reinfeç̧̋̃es e para o controle do surto em Bauru.

As características particulares dos surtos de malária entre usuários de drogas parecem indicar a necessidade de que uma intervenção ativa para seu controle seja adotada. Em Bauru, a simples divulgação do problema junto aos serviços médicos não resultou, por si só, na identificação de novos casos suspeitos. Dos 21 casos ocorridos apenas 5 procuraram os serviços de saúde locais sendo os demais detectados através de visitas domiciliares.

A condição mais importante para o êxito das ações de controle adotadas foi a confiança estabelecida entre os profissionais de saúde destacadas para o trabalho e os casos suspeitos. O modo de abordar o problema e a garantia do sigilo sobre as informações obtidas foram fundamentais para o 
estabelecimento dessa confiança. Em diversas ocasiōes obteve-se a participação ativa dos envolvidos na identificação dos demais suspeitos. Nas raras situações em que essa confiança foi abalada, a colaboração cessou por completo, dificultando muito o desenvolvimento das ações.

Várias dificuldades surgiram durante o trabalho realizado em Bauru, em razão do despreparo da SUCEN e dos serviços de saúde locais para enfrentar situações semelhantes. É possível que, em razăo dessas dificuldades, nem todos os comunicantes tenham sido identificados, o que poderia explicar a ocorrência de novos casos na cidade, a partir de 1991.

Os vários casos de malária detectados no Estado de São Paulo, entre usuários de drogas injetáveis, a partir de 1988, apontam para o possível risco de que a doença se torne endêmica nesses grupos de indivíduos se medidas eficazes de controle não forem adotadas. Em Nova Iorque, durante as décadas de 30 e 40 , a malária era bem conhecida entre os usuários de heroína, que costumavam se automedicar com quinino ${ }^{10}$. Embora essa prática não tenha sido reconhecida até o momento em São Paulo, é possível que ela venha a acontecer, o que resultará em dificuldades adicionais para a avaliação e o controle do problema.

O risco da ocorrência de surtos de transmissão natural de malária em áreas com presença de anofelinos vetores, a partir da presença de usuários de drogas infectados com gametócitos, já foi assinalada por diversos autores $3,6,10,11$. Essa possibilidade, entretanto, parece pouco provável no Estado de São Paulo, uma vez que usuários de drogas são mais comuns nas cidades de médio e grande porte, onde a presença de vetores é rara, se é que exista na maior parte delas. $\mathrm{Na}$ região Amazônia, ao contrário, a presença de vetores nas áreas urbanas é comum e surtos de malária costumam ocorrer, especialmente nos bairros periféricos. Nessas áreas, a presença de usuários de drogas infectados pode resultar em risco adicional para a ocorrência de surtos de transmissão natural da doença.

Os sintomas clínicos da malária induzida não são diferentes daquela naturalmente adquirida, mas podem ser confundidas com crises de abstinência, uso de drogas adulteradas ou com outras doenças comuns entre usuários de drogas ${ }^{3}, 7,10,11$. Nos casos confirmados, observados em Bauru, a maioria apresentou sintomologia própria da infecção por $P$. vivax, com acessos febris característicos a cada 24 horas.

Nos casos de malária por infecção natural diagnosticados pela SUCEN, o intervalo de tempo transcorrido entre o início dos sintomas e o diagnóstico tem sido de 1 a 3 dias em cerca de $50 \%$ deles ${ }^{16}$. Dentre os casos confirmados em Bauru, esse intervalo foi superior a 30 dias, em pelo menos $16(76 \%)$ deles. Evidentemente, se o surto fosse de malária falciparum, numerosas fatalidades teriam sido registradas antes que a situação tivesse sido controlada.

Considerando os recentes casos observados no Estado de São Paulo, a malária deve ser considerada, a partir de agora, como hipótese diagnóstica em todos os indivíduos usuários de drogas endovenosa com febre de origem indeterminada. Nas cidades onde surtos recentes tenham ocorrido, a realização de exames hemoscópicos específicos deve ser considerada também para os usuários de drogas que se acham assintomáticos.

Os serviços de saúde mental que atendem usuários de droga injetável e de assistência à AIDS, especialmente, devem estar permanentemente informados e alertados para o problema.

Finalmente, o presente trabalho mostrou, também, a ocorrência elevada de outras doenças infecciosas de transmissão parenteral e sexual, entre os usuários de drogas. Embora o percentual de exames positivos para sífilis possa refletir apenas a elevada ocorrência de testes VDLR ${ }^{14}$ fạlso positivos entre os usuários de drogas injetáveis ou entre pessoas com malária recente, a frequiência de exames sorológicos positivos para a AIDS e hepatite $B$ indicam a gravidade do problema e a necessidade de adoção de medidas específicas voltadas para a redução da prática do uso de drogas com compartilhamento de seringas e agulhas.

BARATA, L. C. B. et al. [Outbreak of malaria among injectable-drug users]. Rev. Saúde Pública, 27: 9-14, 1993. Five cases of malaria were detected among cocaine users by the local health service in Bauru, a city with a population of 260,000 , located $324 \mathrm{~km}$ from $S$. Paulo, Brazil, during the first three weeks of July 1990. Autochthonous malaria had not occurred in Bauru since 1978 , and all the five cases denied having recently traveled to endemic areas. An extensive epidemiologic survey conducted from July 19 to September 13 revealed that the 5 cases were in fact part of a malaria outbreak among endovenous drug users. Moreover, at least 114 other people, who had in the last three months shared syringes and needles with one or more proved cases, were also involved in the outbreak. One hundred and two people were identified and interviewed orally. The examination of thick smears made from samples collected from 99 of them confirmed 21 cases of vivax malaria. Three people with negative blood smears had an indirect imunofluorescent assay positive to $P$. vivax antigen. Although the index case could not be discovered, the investigation suggested that one or more people who had recently traveled to the Amazon region might have introduced the disease. The control of the outbreak was achieved after offering treatment with chloroquine (10 tablets) to confirmed cases and contacts, followed by weekly suppresive chloroquine ( 2 tablets) until the last contact was detect- 
ed. Malaria examination of 91 blood samples also revealed a high prevalence of hepatitis $B$ virus infection (40\%) and HIV infection (58\%) among those involved in the outbreak. The strategy used to identify the cases and the contacts and the difficulties overcome in carrying it through are described. It is also suggested that malaria might become an endemic disease among endovenous drug users in the State of S. Paulo.

Keywords: Malaria, epidemiology. Plasmodium vivax. Substance abuse, intravenous, complications. Needle sharing.

\section{Agradecimentos}

Aos pesquisadores Ester de Carvalho e Rosa Maria Tubak, da SUCEN, e às seguintes instituiçð̃es: Diretoria de Zoonoses do Centro de Vigilância Epidemiológica da Secretaria de Estado da Saúde, Vigilância Epidemiológica do ERSA e da Prefeitura de Bauru, Instituto Adolfo Lutz, Centro de Referência para Tratamento de AIDS e o Setor Regional de Bauru da SUCEN, pela colaboração prestada.

\section{Referéncias Blbllográficas}

1. BARATA, L. C. B. et al. Nota sobre surto de malária em usuários de drogas injetáveis. Rev. Saúde Pública, 24; 321-22, 1990.

2. BIGGAM, A. G. Malignant malaria associated with the administration of heroin intravenously. Trans. $R$. Soc. Trop. Med. Hyg., 23: 147-53, 1929.

3. DONALD, O. L. et al. Malaria among heroin users. Health Serv. Rep., 87: 545-49, 1972.
4. EATON, L. M. \& FLIMBERG, S. M. Accidental hypodermic transmission of malaria in drug addicts. Am. $J$. Med. Sci., 186: 679-83, 1983.

5. FRIEDMAM, C. T. H. et al. A malaria epidemic among heroin users. Am. J. Med. Hyg., 22: 302-7, 1973.

6. GEIGER, J. C. Malaria in narcotic addicts. JAMA, 98: 1494, 1932.

7. Keams, O. A. et al. Induced malaria-California. $M M W R, 20$ : 99-100, 1971.

8. LO, S. S. et al. Malária induzida por drogas injetáveis associada à soropositividade para HIV em Presidente Prudente - SP. Rev. Saúde Pública, 25: 17-22, 1991.

9. MARQUES, A. C. Human migration and the spread of malaria in Brazil. Parasitol. Today, 25: 17-22, 1991.

10. MOST, H. Falciparum malaria among drug addicts. Am. J. Public. Health, 30: 403-10, 1940.

11. ROSEMBLATT, J. E. \& MARSH, V. H. Induced malaria in narcotic additcs. Lancet, 2: 189-90, 1971.

12. SECRETARIA DE ESTADO DE SAUDE. Centro de Referência e Tratamento de AIDS. Bol. Epidemiol. (dez), 1991.

13. SUPERINTENDENCIA DE CAMPANHAS DE SAUDE PÚBLICA. Fundação Nacional de Saúde. Divisão de Malária. Inf. Téc., (1) 1991.

14 SPARLING, P. F. Diagnosis and treatment of syphilis. $N$. Engl.J. Med., 284: 642-53, 1971.

15. TAUII, P. L. Malária: agrava-se o quadro da doença no Brasil. Ciênc. Hoje, 2(12): 58-64, 1984.

16. WANDERLEY, D. M. V. et al. Malária no Estado de São Paulo: avaliação de aspectos de vigilância epidemiológica. Cad. Saúde Pública, 5: 296-304, 1989.

17. WICH are the apropriated modifications of existing regulations designed to prevent the transmission of malaria by blood transfusion, in view of the increasing frequency of travel to endemic areas? (Intemational Forum) Vox Sang, 52: 138-48, 1987.

Recebido para publicaçäo em 25.6.1992 Reapresentado em 8.10.1992 Aprovado para publicaçăo em 22.10.1992 